\title{
Antisense Oligonucleotides Targeting Influenza A Segment 8 Genomic RNA Inhibit Viral Replication
}

\author{
Elzbieta Lenartowicz, Aitor Nogales, Elzbieta Kierzek, ${ }^{3}$ Ryszard Kierzek, \\ Luis Martínez-Sobrido, and Douglas H. Turner ${ }^{1}$
}

Influenza A virus (IAV) affects 5\%-10\% of the world's population every year. Through genome changes, many IAV strains develop resistance to currently available anti-influenza therapeutics. Therefore, there is an urgent need to find new targets for therapeutics against this important human respiratory pathogen. In this study, $2^{\prime}-O$-methyl and locked nucleic acid antisense oligonucleotides (ASOs) were designed to target internal regions of influenza A/California/04/ 2009 (H1N1) genomic viral RNA segment 8 (vRNA8) based on a base-pairing model of vRNA8. Ten of 14 tested ASOs showed inhibition of viral replication in Madin-Darby canine kidney cells. The best five ASOs were 11-15 nucleotides long and showed inhibition ranging from 5- to 25-fold. In a cell viability assay they showed no cytotoxicity. The same five ASOs also showed no inhibition of influenza B/Brisbane/60/2008 (Victoria lineage), indicating that they are sequence specific for IAV. Moreover, combinations of ASOs slightly improved anti-influenza activity. These studies establish the accessibility of IAV vRNA for ASOs in regions other than the panhandle formed between the $5^{\prime}$ and $3^{\prime}$ ends. Thus, these regions can provide targets for the development of novel IAV antiviral approaches.

Keywords: influenza RNA, antisense oligonucleotides, genomIc RNA

\section{Introduction}

$\mathbf{S}$ EASONAl INFluenza A virus (IAV) causes disease in humans every year, especially in the winter months in temperate climates [1]. The World Health Organization (WHO) estimates that each year, influenza affects 5\%-10\% of the world's population resulting in between 250,000 and 500,000 deaths [2]. Furthermore, IAV occasionally and unpredictably can cause a pandemic when a novel virus not previously circulating in humans is introduced into humans and is easily transmitted person to person [3-5].

IAVs are categorized by the antigenic variation of the hemagglutinin (HA) and neuraminidase (NA) proteins. So far, $16 \mathrm{HA}$ and 9 NA IAV variants have been found in wild birds [6,7]. Only two subtypes, H3N2 and $\mathrm{H} 1 \mathrm{~N} 1$, are currently circulating in humans [8]. The last novel pandemic strain (pH1N1) of IAV appeared in 2009 and became, since then, the dominant H1N1 virus circulating worldwide [9]. In addition, zoonoses can occur, as evidenced by the current outbreaks of H7N9 and H5N1, which may contain pandemic potential of acquiring the ability to readily transmit between humans [10,11].

The IAV genome has eight negative-sense singlestranded RNAs [12,13] that encode for 10-14 proteins
[14]. To date, only two classes of anti-influenza therapeutics are available: (1) Adamantanes (amantadine and rimantadine) target the ion channel matrix 2 (M2) protein $[2,15]$, and (2) inhibitors of influenza NA (zanamivir, oseltamivir as well as peramivir and laninamivir licensed in several countries) $[2,16,17]$. Most circulating IAVs are resistant to amantadine [18], and emerging resistance to zanamivir and oseltamivir has become a serious problem $[9,19,20]$. The rate of oseltamivir-resistant H1N1 influenza viruses in the United States has increased from $0.7 \%$ in the 2006-2007 influenza season to $98.5 \%$ in the 2008-2009 influenza season [21,22]. Therefore, there is an urgent need to find new targets and therapeutics for the treatment of influenza viral infections [23].

Influenza RNA is a potential target for therapeutics. Antisense oligonucleotides (ASOs) provide an approach for identifying potential targets, and therefore represent potential therapeutics. Current ASO therapeutics, including Vitraviren [24] and Mipomersen [25], target mRNA. In this study, ASOs were designed against loops in IAV segment 8 vRNA and tested in cell culture. The results suggest that IAV vRNA loops can be targets for therapeutics, including ASOs.

\footnotetext{
${ }^{1}$ Department of Chemistry, University of Rochester, Rochester, New York.

${ }^{2}$ Department of Microbiology and Immunology, University of Rochester, Rochester, New York.

${ }^{3}$ Institute of Bioorganic Chemistry, Polish Academy of Sciences, Poznan, Poland.
}

(c) Elzbieta Lenartowicz et al., 2016; Published by Mary Ann Liebert, Inc. This Open Access article is distributed under the terms of the Creative Commons Attribution Noncommercial License (http://creativecommons.org/licenses/by-nc/4.0/) which permits any noncommercial use, distribution, and reproduction in any medium, provided the original author(s) and the source are credited. 


\section{Materials and Methods}

\section{Predicted folding of vRNA8 of A/California/04/2009 (H1N1)}

The vRNA8 of influenza A/California/04/2009 (H1N1) was folded by comparison to vRNA8 of A/Vietnam/1203/ 2004 (H5N1) with the Dynalign program [26] in the RNAstructure 5.4 package of software. Dynalign finds the lowest free energy sequence alignment and secondary structure common to two previously unaligned sequences. Folding was guided by the base pairing determined on the basis of chemical mapping and base pair conservation of influenza A/Vietnam/1203/2004 (H5N1) [27]. Base pairs in vRNA8 of A/Vietnam/1203/2004 (H5N1) that are more than $99.9 \%$ conserved in a database of over 8,000 nonredundant IAV sequences were used in Dynalign as constraints when submitting the sequence of vRNA8 of A/Vietnam/1203/ 2004 (H5N1). The pairing from nucleotides 713-718/783-790 was adjusted to maximize alignment of base pairs.

\section{Design and synthesis of ASOs}

ASOs were designed based on the predicted self-folding of protein-free vRNA8 of influenza A/California/04/2009 (H1N1). Oligonucleotides were designed to target singlestranded regions that bind to microarray probes and/or are well defined as single stranded based on chemical mapping of vRNA8 of A/Vietnam/1203/2004 (H5N1) (Supplementary Fig. S1; Supplementary Data are available online at www .liebertpub.com/nat) [27]. All oligonucleotides were primarily 2'-O-methyl RNA with some also containing locked nucleic acid (LNA) nucleotides to increase duplex stability. All oligonucleotides were synthesized on MerMade 12 (BioAutomation) synthesizer by phosphoroamidite method and deprotected according to published protocols [28,29]. Oligonucleotides were purified with thin layer chromatography or with denaturing $12 \%$ polyacrylamide gel and concentrations were measured with a UV spectrophotometer (NanoDrop—-Thermo Scientific).

\section{Cells and viruses}

Madin-Darby canine kidney (MDCK) cells (ATCC CCL34) were maintained in Dulbecco's modified Eagle's medium (DMEM) supplemented with 10\% fetal bovine serum (FBS) and $1 \%$ PSG (penicillin-100 U/mL, streptomycin-100 $\mu \mathrm{g}$ / $\mathrm{mL}, \mathrm{L}$-glutamine $-2 \mathrm{mM}$ ) at $37^{\circ} \mathrm{C}$ in air enriched with $5 \% \mathrm{CO}_{2}$.

Influenza A/California/04/2009 (H1N1) and influenza B/ Brisbane/60/2008 (Victoria lineage) were propagated in MDCK cells and viral titers were measured with standard plaque assays [plaque-forming units (PFU)/mL]. Briefly, confluent monolayers of MDCK cells (six-well plate format) were infected with 10-fold viral dilutions for $1 \mathrm{~h}$ at room temperature (RT), overlaid with agar, and incubated at $33^{\circ} \mathrm{C}$ in air enriched with $5 \% \mathrm{CO}_{2}$. After 3 days postinfection, cells were fixed with $4 \%$ formaldehyde in phosphate-buffered saline (PBS), and the agar layer was removed. One percent crystal violet was used to visualize the viral plaques. Virus stocks consisted of cellfree supernatant and were kept in aliquots at $-80^{\circ} \mathrm{C}$.

\section{MDCK cell transfections}

Lipofectamine $^{\circledR} 2000$ (Invitrogen) was used as a lipidbased carrier. One day before transfection, $2.5 \times 10^{6}$ cells were seeded in a $100-\mathrm{mm}$ dish to be sure the cells would be in the exponential growth phase on the day of transfection.
Before each transfection, Lipofectamine 2000 was diluted 10 -fold with the Opti-MEM ${ }^{\circledR}$ I Reduced Serum Medium (Opti-MEM) and incubated $10 \mathrm{~min}$ at RT. Then $15 \mu \mathrm{L}$ of this solution was mixed gently with $15 \mu \mathrm{L}$ of $5 \mu \mathrm{M}$ ASO in OptiMEM and incubated $30 \mathrm{~min}$ at RT. Next, $300 \mu \mathrm{L}$ of $7.5 \times 10^{4}$ MDCK cells in media was added. For each ASO, $100 \mu \mathrm{L}$ of this mixture was added to each of three wells in a 96-well plate (Supplementary Fig. S2A). Cells were incubated at $37^{\circ} \mathrm{C}$ in air enriched with $5 \% \mathrm{CO}_{2}$ for $6 \mathrm{~h}$ and the medium was changed for fresh one. Transfection efficiency was optimized using FAM6-labeled oligonucleotide (FN) observed under a fluorescent microscope (Supplementary Fig. S2B).

Concentrations of oligonucleotide from 0.01 to $2 \mu \mathrm{M}$ (final concentration in the medium) and different ratios of oligonucleotide to Lipofectamine 2000 ( $\mu \mathrm{M}$ oligonucleotide to $\mu \mathrm{L}$ Lipofectamine $2000=1: 0.5 ; 1: 1 ; 1: 1.5 ; 1: 2 ; 1: 2.5)$ were tested. Using a fluorescent microscope, FN was observed to accumulate in both the nucleus and cytoplasm (Supplementary Fig. S2B). The optimal condition for uptake was chosen as the ratio of oligonucleotide to Lipofectamine $2000=1: 2$ (Supplementary Fig. S2). According to Invitrogen, the toxicity of undiluted Lipofectamine 2000 is minimized at $0.5 \mu \mathrm{L} /$ well in a 96-well plate. Accordingly, the maximum concentration of oligonucleotides used in this study was $230 \mathrm{nM}$. Transfections of all ASOs were performed in triplicate.

\section{Antiviral test of ASOs}

At $18 \mathrm{~h}$ posttransfection with ASO (6h with liposome complexes and $12 \mathrm{~h}$ with fresh medium, as described above), MDCK cells were infected (multiplicity of infection, of 0.001) with the indicated viruses diluted in PBS supplemented with $0.3 \%$ bovine albumin (BA) and 1\% PS (PBS/ $\mathrm{BA} / \mathrm{PS})$. After $1 \mathrm{~h}$ incubation at $\mathrm{RT}$ on a rocking platform, the infection medium was changed for the postinfection medium containing DMEM supplemented with $0.3 \%$ BA, $1 \%$ PSG, and $1 \mu \mathrm{g} / \mathrm{mL}$ tosyl-sulfonyl phenylalanyl chloromethyl ketone (TPCK)-treated trypsin (Sigma). Infected MDCK cells were incubated at $33^{\circ} \mathrm{C}$ in air enriched with $5 \% \mathrm{CO}_{2}$ for $36 \mathrm{~h}$ and after that time, cell culture supernatants (CCS) were collected. Viral titers were determined with the immunofocus assay described below.

\section{Immunofocus assay}

CCS were 10-fold diluted in PBS/BA/PS. MDCK cells cultured on $96-$ well plates were infected with $50 \mu \mathrm{L}$ of CCS dilutions for $1 \mathrm{~h}$ at RT. After incubation, the infection medium was changed for the postinfection medium and infected cells were maintained at $33^{\circ} \mathrm{C}$ in air enriched with $5 \% \mathrm{CO}_{2}$ for $8-10 \mathrm{~h}$. Cells were fixed with $4 \%$ paraformaldehyde (PFA) for $15 \mathrm{~min}$ and permeabilized with $0.5 \%$ Triton X-100 in PBS for $15 \mathrm{~min}$ at RT. Next, cells were incubated with a blocking buffer containing $2 \%$ bovine serum albumin in PBS for $1 \mathrm{~h}$. After blocking, the solution was replaced with a mouse anti-NP (HB-65 for IAV or B017 for IBV) primary monoclonal antibody diluted in the blocking buffer $(1 \mu \mathrm{g} / \mathrm{mL})$ and incubated for $1 \mathrm{~h}$ at $37^{\circ} \mathrm{C}$. Primary antibodies were detected with an FITC-conjugated rabbit anti-mouse polyclonal secondary antibody (Dako 1:200). Fluorescent-forming units (FFU/mL) were counted under a fluorescent microscope. The mean titer from triplicates and the standard deviation (SD) were calculated with Microsoft Excel software. 


\section{Cell viability assays}

For the cell viability assays, MDCK cells were transfected as described above. The medium containing liposome complexes was changed for fresh one after $6 \mathrm{~h}$ and after $18 \mathrm{~h}$, cell viability was measured using the CellTiter $96^{\circledR}$ Non-Radioactive Cell Proliferation Assay (MTT; Promega) according to the manufacturer's recommendations. The rate of formazan dye formation was determined by measuring the absorbance (570$650 \mathrm{~nm})$. The $570-650 \mathrm{~nm}$ reading value is directly proportional to the number of living cells. Cell viability was normalized to viability of cells treated only with Lipofectamine 2000. The mean and SD from triplicates were calculated with Microsoft Excel software.

\section{Statistical analysis}

Experimental data were analyzed with a two-tailed $t$-test with unequal variance in Microsoft Excel Software. Three intervals of statistical confidence were considered, 0.05, 0.01, and 0.001. The statistic was calculated from three independent experiments with each containing three technical repeats, normalized to virus titer from cells treated with Lipofectamine 2000 only.

\section{Results}

\section{Design of ASOs}

Self-folding of protein-free vRNA8 from A/Vietnam/1203/ 2004 (H5N1) [27] was used to model A/California/04/2009 (H1N1), which is more representative of influenza strains
(H1N1) currently circulating in humans. The sequences are 83\% homologous and the predicted secondary structures are similar (Fig. 1 and Supplementary Fig. S1). Single-stranded regions in A/Vietnam/1203/2004 (H5N1) vRNA8 that bind to microarray probes and are well defined by chemical mapping [27] were chosen as targets (Fig. 1 and Supplementary Fig. S1). All designed ASOs were fully complementary to vRNA8 of A/ California/04/2009 (H1N1) (Fig. 1 and Table 1). With the exception of ASOs 276-20M \& L, 713-10M, and 786-19M, the length of ASOs was dependent on the length of loops and sometimes adjacent pairs with low probability based on results for A/Vietnam/1203/2004 (H5N1) (Fig. 1 and Supplementary Fig. S1) [30]. ASOs 276-20M \& L target a hairpin with a stem of highly probable base pairs and ASOs 713-10M and 786-19M also target a helical region with highly probable base pairs in $\mathrm{A} /$ Vietnam/1203/2004 (H5N1). In addition, a sequence with random A, G, and U (5'GAGGAGUGUAGAGUUAUA) without (NEG) and with (FN) a 5'FAM fluorophore was also used as a negative control. All ASOs were primarily 2 - $O$-methyl RNA with half also containing LNA nucleotides to stabilize binding to target sequence (Table 1).

\section{Certain ASOs inhibit IAV propagation.}

ASOs at final total concentration of $230 \mathrm{nM}$ were tested in MDCK cells (Supplementary Fig. S2). Ten of 14 tested ASOs showed significant $(P<0.05)$ inhibition of IAV replication (Fig. 2A). Five ASOs inhibited virus propagation more than fivefold at $36 \mathrm{~h}$ postinfection. ASOs $187-14 \mathrm{~L}$ and $404-14 \mathrm{~L}$

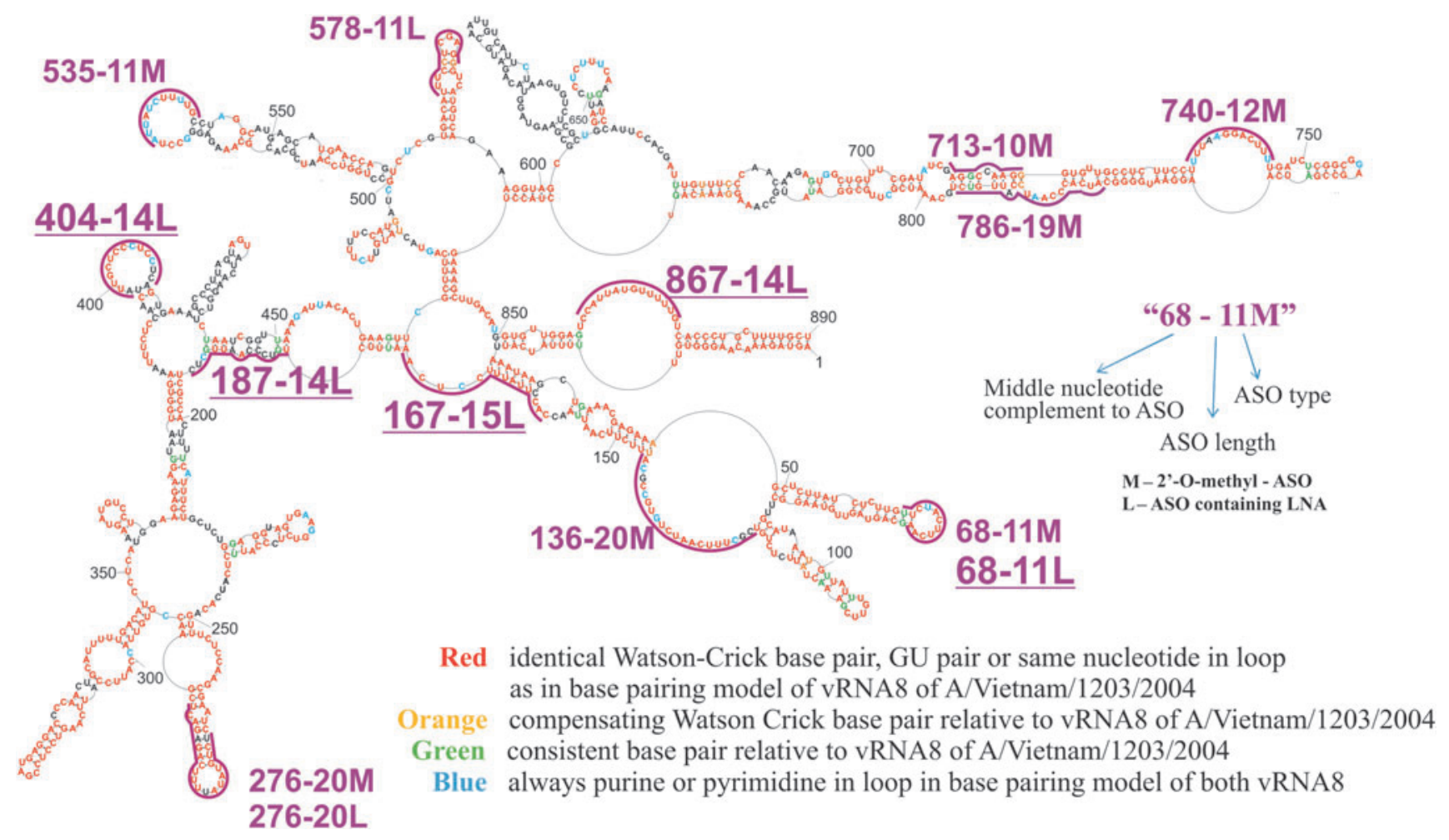

FIG. 1. Targets of ASOs in predicted secondary structure of vRNA8 of influenza A/California/04/2009 (H1N1). Purple lines indicate regions targeted by ASOs. The nomenclature consists of vRNA8 nucleotide number complementary to the middle nucleotide of ASOs, length of ASOs, and containing modification: $\mathrm{M}=2^{\prime}-O$-methyl-RNA and L $=2^{\prime}-O$-methyl-RNA with LNA. Note that relative to influenza A/Vietnam/1203/2004 (H5N1) (Supplementary Fig. S1) there is a 15 nucleotide insert between 614 and 615 so that the nucleotide numbers after 615 are larger by 15 relative to those for A/Vietnam/1203/2004 (H5N1). The numbering of vRNA8 is from its $5^{\prime}$ end. A consistent base pair is one where GU replaces a Watson-Crick pair or vice versa. 
Table 1. Sequences of ASOs Fully Complementary to vRNA8 of Influenza A/California/04/2009 (H1N1)

\begin{tabular}{|c|c|c|c|c|c|c|}
\hline \multirow[b]{2}{*}{ Name } & \multirow[b]{2}{*}{ Sequence $\left(5^{\prime}-3^{\prime}\right)^{\mathrm{a}}$} & \multirow{2}{*}{$\frac{\Delta G_{37}^{\circ}(\mathrm{kcal} / \mathrm{mol})}{\text { Duplex }^{\mathrm{b}}}$} & \multirow{2}{*}{$\frac{\Delta G_{37}^{\circ}(\mathrm{kcal} / \mathrm{mol})}{v R N A 8 \text { binding }^{\mathrm{c}}}$} & \multirow{2}{*}{$\begin{array}{c}\text { Predicted } \\
K_{d}\end{array}$} & \multirow{2}{*}{$\begin{array}{c}\log _{10} \\
\text { inhibition }\end{array}$} & \multirow{2}{*}{$\begin{array}{c}\text { Percent } \\
\text { of inhibition }\end{array}$} \\
\hline & & & & & & \\
\hline 404-14L & $\mathrm{GA}^{\mathrm{L}} \mathrm{GGA}^{\mathrm{L}} \mathrm{GGGA}^{\mathrm{L}} \mathrm{GCA}^{\mathrm{L}} \mathrm{AU}$ & -32.9 & -32.9 & 7.1E-24 & 1.4 & 96.2 \\
\hline $187-14 \mathrm{~L}$ & $\mathrm{GA}^{\mathrm{L}} \mathrm{GCA}^{\mathrm{L}} \mathrm{AU}^{\mathrm{L}} \mathrm{UGGGA}^{\mathrm{L}} \mathrm{CA}$ & -30.8 & -30.6 & $2.9 \mathrm{E}-22$ & 1.4 & 96.0 \\
\hline $68-11 \mathrm{~L}$ & $U^{\mathrm{L}} \mathrm{GAA}^{\mathrm{L}} \mathrm{GU}^{\mathrm{L}} \mathrm{GGA}^{\mathrm{L}} \mathrm{A}$ & -22.1 & -18.7 & 7.0E-14 & 1.1 & 92.1 \\
\hline $167-15 \mathrm{~L}$ & $\mathrm{UGA}^{\mathrm{L}} \mathrm{GGA}^{\mathrm{L}} \mathrm{AAU}^{\mathrm{L}} \mathrm{AAGGU}^{\mathrm{L}} \mathrm{G}$ & -30.1 & -27.7 & $3.1 \mathrm{E}-20$ & 0.8 & 84.1 \\
\hline $867-14 \mathrm{~L}$ & $\mathrm{CA}^{\mathrm{L}} \mathrm{AA}^{\mathrm{L}} \mathrm{AA}^{\mathrm{L}} \mathrm{CA}^{\mathrm{L}} \mathrm{UA}^{\mathrm{L}} \mathrm{AUGG}$ & -24.0 & -24.0 & $1.2 \mathrm{E}-17$ & 0.7 & 80.0 \\
\hline 136-20M & AUGCGGCACAGAUUGAAAGC & -38.5 & -38.5 & 7.2E-28 & 0.5 & 68.4 \\
\hline $276-20 \mathrm{~L}$ & AGU $^{\mathrm{L}} \mathrm{CUC}^{\mathrm{L}} \mathrm{UGAA}^{\mathrm{L}}$ AAUA ${ }^{\mathrm{L}} \mathrm{UACA}^{\mathrm{L}} \mathrm{GA}$ & -39.2 & -25.6 & $9.4 \mathrm{E}-19$ & 0.5 & 68.4 \\
\hline $68-11 \mathrm{M}$ & UUGAAGUGGAA & -17.0 & -13.6 & $2.8 \mathrm{E}-10$ & 0.4 & 60.2 \\
\hline $535-11 \mathrm{M}$ & CAAAAGAUAAU & -11.2 & -11.2 & $1.4 \mathrm{E}-08$ & 0.2 & 36.9 \\
\hline $578-11 \mathrm{~L}$ & $\mathrm{CCU}^{\mathrm{L}} \mathrm{CGA}^{\mathrm{L}} \mathrm{GGA}^{\mathrm{L}} \mathrm{AA}$ & -23.3 & -17.0 & $1.1 \mathrm{E}-12$ & 0.2 & 36.9 \\
\hline $740-12 \mathrm{M}$ & AAAGUCCUUAAA & -15.6 & -15.6 & $9.8 \mathrm{E}-12$ & 0.2 & 36.9 \\
\hline $276-20 \mathrm{M}$ & AGUCUCUGAAAAUAUACAGA & -32.5 & -18.9 & $5.0 \mathrm{E}-14$ & 0.1 & 20.6 \\
\hline 713-10M & CCUUGGCCUC & -20.7 & -11.8 & 5.0E-09 & 0.0 & 0.0 \\
\hline 786-19M & GACAAUGGAUUGGGUGAUG & -35.8 & -15.1 & $2.2 \mathrm{E}-11$ & 0.0 & 0.0 \\
\hline $\mathrm{FN}$ & FAM6-GAGGAGUGUAGAGUUAUA & - & & & -0.1 & -25.9 \\
\hline NEG & GAGGAGUGUAGAGUUAUA & - & & & -0.2 & -58.5 \\
\hline
\end{tabular}

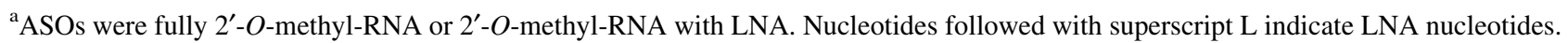
NEG is a scrambled oligonucleotide, not complementary to any sequence of vRNAs of influenza A/California/04/2009 (H1N1). FNscrambled oligonucleotide labeled with FAM6 at the $5^{\prime}$ end, used to test transfection efficiency.

${ }^{\text {b} P r e d i c t e d ~} \Delta \mathrm{G}_{37}^{\circ}$ of the ASO binding to a complementary single-stranded RNA. Predicted free energy for duplex was calculated with 2'OMeRNA/RNA or LNA-2'OMeRNA/RNA calculator available at www.ibch.poznan.pl/laboratory-of-rna-chemistry.

${ }^{\mathrm{c}}$ Predicted $\Delta \mathrm{G}^{\circ}{ }_{37}$ for ASO binding to vRNA8. The $\Delta \mathrm{G}^{\circ}{ }_{37}$ was calculated by adding to $\Delta \mathrm{G}_{37}^{\circ}$ of duplex formation the $\Delta \mathrm{G}^{\circ}{ }_{37}$ predicted with OligoWalk [50] for breaking base pairs in the region targeted. For binding of oligonucleotides to large loops, no structure needed to be broken.

ASO, antisense oligonucleotide; LNA, locked nucleic acid.

showed the highest inhibitory effect, inhibiting virus propagation on average by 25 -fold $\left(\log _{10} 25=1.4\right)$. Results for all 14 ASOs are listed in Table 1 and shown in Fig. 2A.

Further studies were focused on the five ASOs with more than threefold inhibition of viral propagation $(68-11 \mathrm{~L}, 167-$ 15L, 187-14L, 404-14L, and 867-14L). In these experiments, ASO 578-11L was used as a negative control because it has three LNAs and a predicted $\mathrm{K}_{\mathrm{d}}$ of $1.1 \times 10^{-12}$.

\section{ASOs do not affect cell viability and are specific to IAV}

Five inhibiting ASOs plus two negative control oligonucleotides, NEG and ASO 578-11L, were tested for cytotoxicity (Supplementary Fig. S3). None showed significant $(P<0.05)$ differences in cell viability at $230 \mathrm{nM}$, suggesting that the inhibitory effect of the ASOs on influenza A/California/04/2009 $\mathrm{H} 1 \mathrm{~N} 1$ viral replication is not due to affecting cell viability, but rather due to specifically targeting vRNA8.

To further test if inhibition of IAV replication is specific, the effect of ASOs on IBV infection was studied (Fig. 2B). The sequences of the IBV strain B/Brisbane/60/2008 used for this test do not contain regions fully complementary to the ASOs. The transfection conditions and infection with IBV were the same as for IAV with one exception. The CCS were collected after $48 \mathrm{~h}$ postinfection because of the slower growth of IBV in MDCK cells [31]. No significant differences in IBV titer were observed between the control and cells treated with ASOs (Fig. 2B). Evidently, the inhibition of IAV by all five tested ASOs is specific to sequences of IAV.

\section{Dose-dependent effect of inhibition on IAV}

Two different concentrations (230 and $115 \mathrm{nM}$ ) of ASOs were used on the same assay and with the same amount of
Lipofectamine 2000 to test for a dose-dependent inhibition effect against influenza A/California/04/2009 (Fig. 3). The procedures were the same as for single concentration studies except for the different ASO concentrations. All five ASOs showed a dose-dependent inhibitory effect against influenza A/California/04/2009. The dose-dependent effect was most significant for the three ASOs with the highest inhibition effect (68-11L, 187-14L, and 404-14L). The inhibition level went from 25 -fold $\left(\log _{10} 25=1.4\right)$ relative to control to sixfold $\left(\log _{10} 6=0.8\right)$ for ASO $404-14 \mathrm{~L}$, from 20 -fold $\left(\log _{10} 20=\right.$ 1.3 ) to fourfold $\left(\log _{10} 4=0.6\right)$ for $68-11 \mathrm{~L}$, and from 16 -fold $\left(\log _{10} 16=1.2\right)$ to eightfold $\left(\log _{10} 8=0.9\right)$ for $187-14 \mathrm{~L}$. Smaller dose effects were observed for 167-15L and 867-14L (Fig. 3). Concentration-dependent differences in inhibition were statistically significant $(P<0.05)$ for $68-11 \mathrm{~L}, 187-14 \mathrm{~L}$, and 404-14L.

\section{Effect of combinations of ASOs on influenza A/California/04/2009 viral replication}

All possible combinations of two, three, and four ASOs with 867-14L were tested for an additive effect against viral replication (Fig. 4). The final total concentration $(230 \mathrm{nM})$ of ASOs in mixtures was the same in all single or combined transfections. Transfection and infection protocols were the same as in single ASO tests. ASO 867-14L tested individually showed average inhibition of fivefold relative to control virus titer. After it was combined with other ASOs, the largest inhibition was 20-fold. The additive effect of ASO combinations was relatively small, but statistically significant differences $(P<0.05)$ were observed in eight cases compared to single ASO 867-14L inhibition (Fig. 4). 


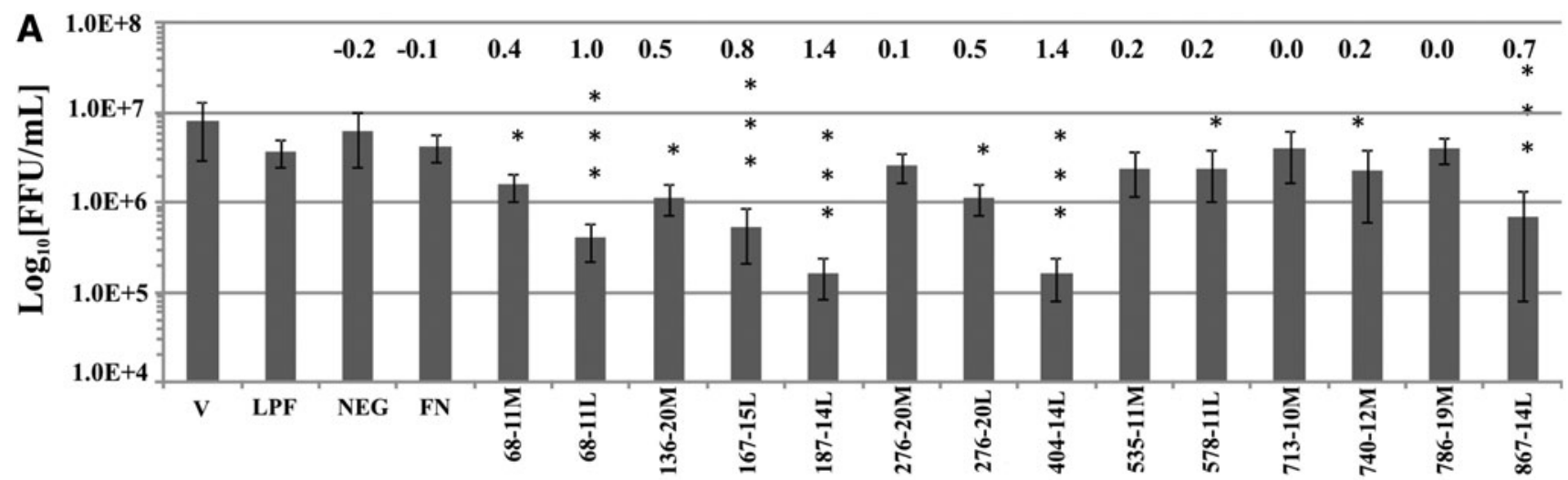

B

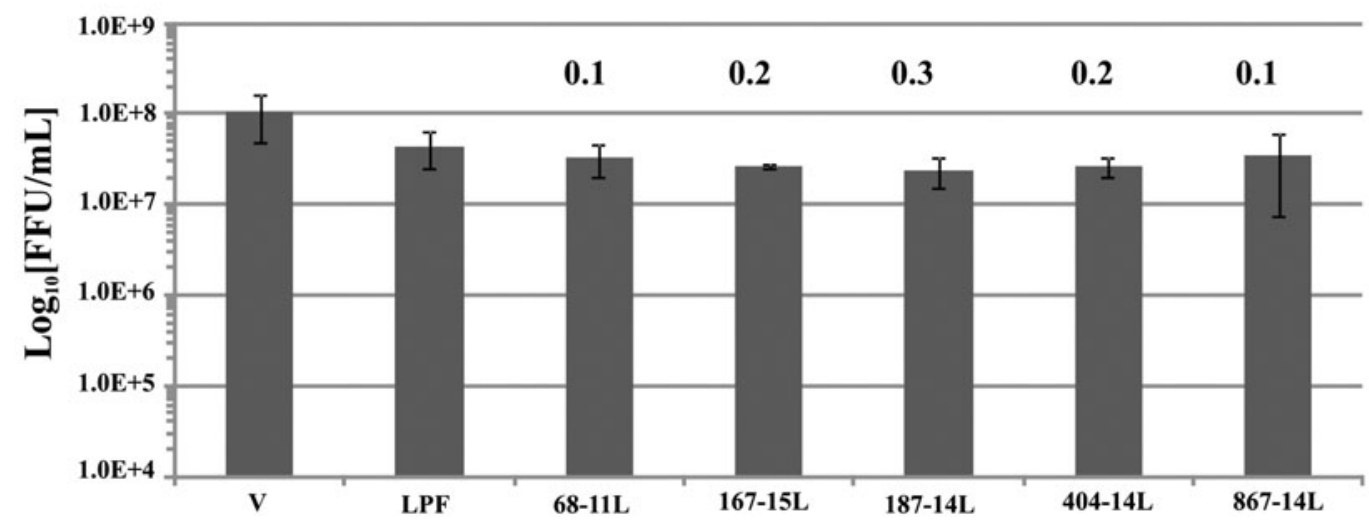

FIG. 2. Antiviral activity of $230 \mathrm{nM}$ ASOs in MDCK cells. Logarithm of average titer of influenza in CCS was analyzed by the immunofocus assay (FFU/mL): (A) for A/California/04/2009 after $36 \mathrm{~h}$ postinfection and (B) for B/Brisbane/60/2008 after $48 \mathrm{~h}$ postinfection. The mean was calculated from three independent experiments each containing three technical repeats. Numbers above bars indicate the $\log _{10}$ differences between control virus titer from cells treated with Lipofectamine $^{\circledR} 2000$ only (LPF) and with particular ASO added. Statistics were calculated using two-tailed T-test $(* P<0.05$; $* * P<0.01 ; * * * P<0.001)$. No statistically significant differences in virus titer were observed for influenza $\mathrm{B} / \mathrm{Brisbane/60/}$ 2008. CCS, cell culture supernatants; MDCK, Madin-Darby canine kidney.

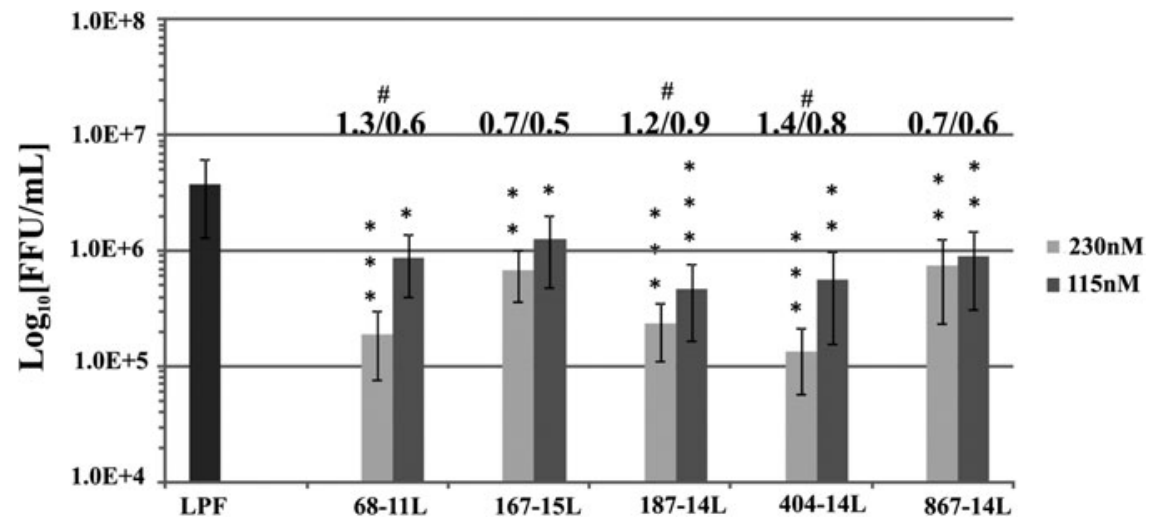

FIG. 3. Dose-dependent antiviral activity of ASOs against influenza A/California/04/2009 (H1N1) in MDCK cells. Logarithm of average titer of influenza A/California/04/2009 (H1N1) in CCS was analyzed after 36h postinfection by immunofocus assay (FFU/mL). The mean was calculated from three independent experiments each containing three technical repeats. In this study, 230 and $115 \mathrm{nM}$ concentrations of oligonucleotides were tested. Numbers above bars indicate the $\log _{10}$ differences between control virus titer from cells treated with Lipofectamine 2000 only (LPF) and with a particular ASO added. Statistics for comparison to LPF and for effect of concentration were calculated using two-tailed Ttest $\left(* P<0.05\right.$; ** $P<0.01$; *** $P<0.001$ for comparison to LPF and ${ }^{\#} P<0.05$ for comparison of concentrations). 


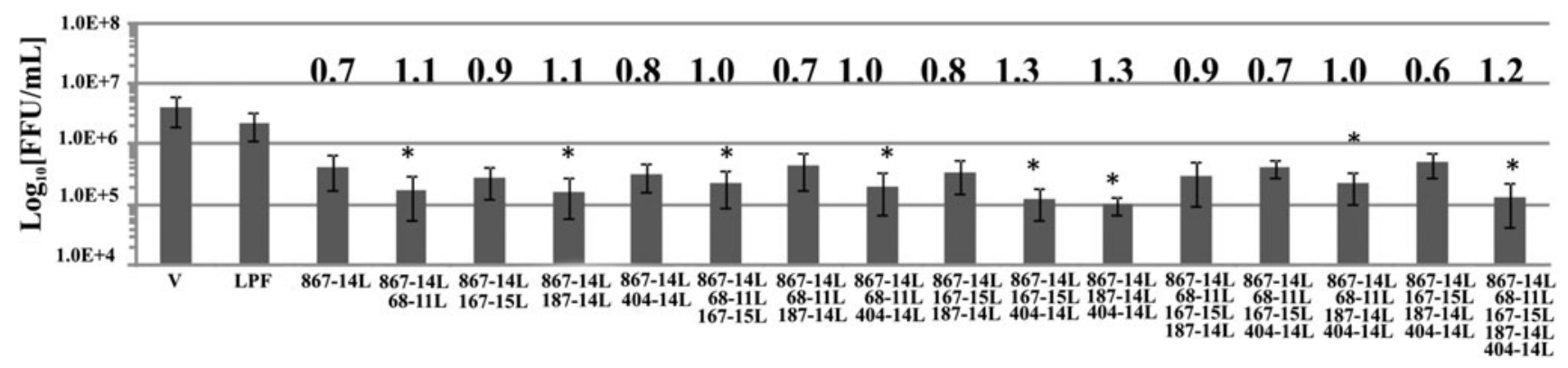

FIG. 4. Antiviral activity of combinations of ASOs against influenza A/California/04/2009 (H1N1) in MDCK cells. Logarithm of average titer of influenza A/California/04/2009 in CCS was analyzed after $36 \mathrm{~h}$ postinfection by immunofocus assay $(\mathrm{FFU} / \mathrm{mL})$. The mean was calculated from three independent experiments each containing three technical repeats. In this study, $230 \mathrm{nM}$ total concentration of oligonucleotides was tested with equal concentration of each ASO. Numbers above bars indicate the $\log _{10}$ differences between control virus titer from cells treated with Lipofectamine 2000 (LPF) only and a particular ASO. Statistics were calculated for differences between $230 \mathrm{nM}$ of $867-14 \mathrm{~L}$ only and $230 \mathrm{nM}$ total ASO concentration for combinations of ASOs using two-tailed T-test $(* P<0.05)$.

\section{LNA can enhance ASO inhibition}

ASOs 68-11 and 276-20 were tested as completely $2^{\prime}-O$ methyl modified RNA and with several LNA substitutions (Figs. 1 and 2; Table 1). For both sequences, inhibition was higher for the ASO containing LNA (Fig. 2A). For ASO 6811 , inhibition increased from 2.5- to 10-fold upon LNA substitution (with $P<0.05$ for $68-11 \mathrm{M}$ and $P<0.001$ for 68 $11 \mathrm{~L})$. For ASO 276-20, no significant inhibition was observed for 276-20M, but a threefold inhibition (with $P<0.05$ ) was observed for 276-20L. These data suggest that LNA substitutions can enhance the inhibitory effect for the designed ASOs, and this would be important to design future ASOs with therapeutic potential against influenza virus targeting vRNA8 or other vRNAs.

\section{Discussion}

IAV yearly infects millions of people and can undergo genetic reassortments to generate a new virus strain for which people do not have preexisting immunity, therefore providing pandemic potential $[32,33]$. Thus, new antiviral therapeutics and targets are urgently needed for the treatment of these newly introduced human viruses against which vaccines and effective antiviral therapies would not be available. In this study, ASOs were designed to target vRNA of segment 8 of influenza A/California/04/2009 (H1N1), the strain responsible for the pandemic of 2009 [34]. All eight influenza vRNA segments are required for efficient virus replication [35]. Thus, inhibition of any segment can have a significant impact on virus propagation.

Most previous ASOs for IAV were designed to target mRNA (e.g., AUG region of different segments [36-40], conserved region [41], or structures [42] in NP, or UTRs $[43,44])$. For IAV vRNA, only the conserved $5^{\prime}$ and $3^{\prime}$ ends forming the panhandle structure have been targeted $[45,46]$. In this study, ASOs were designed to target internal regions in vRNA8, for which function is not yet known. ASO 867-14L, however, targets a region potentially important for influenza virus packaging because deletion of nucleotides 865-875 reduced vRNA8 packaging into virion by over $90 \%[47,48]$.

The ASOs for influenza A/California/04/2009 (H1N1) were designed based on self-folding of vRNA8 of influenza A/Vietnam/1203/2004 (H5N1) [27]. Both strains are impor- tant in IAV studies. Influenza A/California/04/2009 (H1N1) was isolated from humans and caused the pandemic in 2009 [34]. Influenza A/Vietnam/1203/2004 (H5N1) is an avianorigin virus, able to also infect humans [3,49]. So far, no transmission from human to human has been observed with influenza A/Vietnam/1203/2004 (H5N1), but this highly pathogenic strain causes severe disease with high mortality in humans and mutations allowing human to human transmission of the virus could potentially result in a pandemic $[3,50]$. Moreover, it has been shown that influenza A/Indonesia/5/ 2005 (H5N1) can acquire the ability for air transmission between ferrets [50]. Sequences of IAV in A/Vietnam/1203/ 2004 (H5N1) and A/California/04/2009 (H1N1) segment 8 vRNAs are $83 \%$ homologous with $76 \%$ of base pairs conserved (Fig. 1 and Supplementary Fig. S1). Thus, the secondary structure of vRNA8 of influenza A/Vietnam/1203/ 2004 (H5N1) was used to model vRNA8 of influenza A/ California/04/2009 (H1N1) and to select regions to target.

The ASOs generally target predicted single-stranded regions with adjacent stems predicted to be dynamic in proteinfree vRNA8 [27]. Seven of 14 tested ASOs inhibited virus growth by at least threefold in MDCK cells (Table 1). ASOs 68-11M, 276-20M, 535-11M, 578-11L, 713-10M, 740-12M, and $786-19 \mathrm{M}$ had less antiviral activity. The lack of antiviral activity is likely due to relatively weak binding after considering the predicted cost of breaking target structure (Table 1). This is consistent with the enhanced activity of ASO 276-20L, where five LNA nucleotides were introduced. LNA nucleotides strengthen binding affinity [51] and the antiviral activity increased threefold. Moreover, the lack of inhibition by ASOs 713-10M and 786-19M is consistent with the predicted cost of breaking the helix targeted by these ASOs (Fig. 1 and Table 1).

ASOs 68-11L, 187-14L, and 404-14L reduced viral titer more than 10 -fold after $36 \mathrm{~h}$. ASOs $68-11 \mathrm{~L}$ and $404-14 \mathrm{~L}$ primarily target single-stranded nucleotides in a hairpin loop and have predicted tight binding (Table 1). ASO 187-14L also is predicted to bind tightly. Moreover, it binds to a region predicted to have base pairs with low probability (Supplementary Fig. S1) and, therefore, likely to be dynamic. While there are many possible reasons for variation in the activity of ASOs, the results in Table 1 are consistent with previous studies that binding affinity is important [52,53]. 
The results in Table 1 are somewhat surprising. Influenza A genomic vRNA is generally considered not to be a target for ASOs because it is encapsidated by the viral nucleoprotein, NP [54,55]. Moreover, influenza NP binding is expected to disrupt the secondary structure [56]. Nevertheless, when breaking a predicted target structure is considered, with the exception of 276-20M, the ASOs in Table 1 can be categorized in two groups. Those with predicted dissociation constants less than $10^{-13}$ inhibit replication at least $68 \%$, while those with larger dissociation constants inhibit less (Table 1). The exception of $276-20 \mathrm{M}$ may be due to potential selffolding into a hairpin. While factors such as ASO chemical stability, target function, and competition with binding to protein or other RNAs are expected to affect response to ASOs, the results are consistent, with target RNA secondary structure being one factor.

Five ASOs (68-11L, 167-15L, 187-14L, 404-14L, and 867-14L) with more than fivefold inhibition were tested in detail. None are cytotoxic (Supplementary Fig. S3) or inhibit IBV (Fig. 2B), consistent with sequence specificity to IAV. The relatively short lengths of 11-15 nucleotides may reduce possible off-target effects even though predicted dissociation constants are very favorable for binding to the predicted selffolding of the naked vRNA8 [57,58]. ASOs 68-11L, 187-14L, and 404-14L showed statistically significant less inhibition at $115 \mathrm{nM}$ compared to $230 \mathrm{nM}$, but retained the antiviral activity at $115 \mathrm{nM}$ (Fig. 3).

Combinations of ASOs were tested for additive effects (Fig. 4). Of the five ASOs tested extensively, ASO 867-14L alone had the smallest antiviral activity. To see if activity could be enhanced, combinations of other ASOs with 867$14 \mathrm{~L}$ were tested. Statistically significant differences relative to ASO $867-14 \mathrm{~L}$ alone were observed for eight combinations, including the combination of all five tested ASOs. However, the largest enhancement of antiviral activity from combinations of ASOs was only fourfold when total ASO concentration was maintained at $230 \mathrm{nM}$ (Fig. 4). The small additive effect could be because multiple ASOs are not binding to a single vRNA molecule. If a combination of ASOs targeting different segments was used, then the antiviral effect could be higher. Self-folding of other vRNA segments, however, has not been published to facilitate design of new ASOs that could be tested in combination with the ASOs from this study.

There are many possible reasons for the effects of ASOs on influenza. Oligonucleotides accumulate in both cytoplasm and nucleus [59] (Supplementary Fig. S2B). Thus, ASOs can interfere with the virus at every stage of the life cycle. Influenza vRNA is involved in replication, transcription, and packaging. For packaging, there is increasing evidence that vRNA interactions play essential roles in selecting and assembling the required 8 segments into nascent virions [60-62]. For example, vRNA segments can directly interact pairwise in vitro. Packaging of vRNA8 apparently depends on regions corresponding to the $5^{\prime}$ and $3^{\prime}$ UTRs and to part of the open reading frame $[47,48]$. Mutation of this region can result in inhibition of the virus life cycle [63-65]. Based on effects of ASOs, we identify new possible regions for study of vRNA function and thus as potential targets for therapeutics.

In summary, new ASOs with anti-IAV activity are reported in this study. Notably, the antiviral activity was observed when targeting genomic sequences outside the panhandle region, which was previously thought to be the only vRNA region accessible for targeting with ASOs. Correlation between in vitro self-folding of protein-free vRNA8 and the anti-IAV activity suggests that studies of in vitro base pairing can guide ASO design and help identify targets for therapeutics. While only vRNA8 was targeted in this study, it is likely that ASO combinations targeting more than one vRNA would have an even larger anti-viral activity.

\section{Acknowledgments}

We thank Dr. Daniel R. Perez in the Department of Population Health at University of Georgia for the influenza B/ Brisbane/60/2008 virus. This work was supported by NIH grants GM22939 (D.H.T.) and R03TW008739 (D.H.T. and E.K.), as well as National Science Centre grants UMO2013/08/A/ST5/00295 (R.K.) and UMO-2013/08/M/NZ1/ 01062 (E.K.), and by the 2014 University of Rochester Research Award (L.M.-S.).

\section{Author Disclosure Statement}

No competing financial interests exist.

\section{References}

1. Taubenberger JK, AH Reid, TA Janczewski and TG Fanning. (2001). Integrating historical, clinical and molecular genetic data in order to explain the origin and virulence of the 1918 Spanish influenza virus. Philos Trans R Soc Lond B Biol Sci 356:1829-1839.

2. WHO. (2014). Influenza (Seasonal). Fact sheet 211.

3. Taubenberger JK and DM Morens. (2009). Pandemic influenza-including a risk assessment of H5N1. Rev Sci Tech 28:187-202.

4. Parrish CR, PR Murcia and EC Holmes. (2015). Influenza virus reservoirs and intermediate hosts: dogs, horses, and new possibilities for influenza virus exposure of humans. $\mathrm{J}$ Virol 89:2990-2994.

5. Dundon WG, A Heidari, A Fusaro, I Monne, MS Beato, G Cattoli, G Koch, E Starick, IH Brown, et al. (2012). Genetic data from avian influenza and avian paramyxoviruses generated by the European network of excellence (EPIZONE) between 2006 and 2011-review and recommendations for surveillance. Vet Microbiol 154:209-221.

6. Schwartzman LM, AL Cathcart, LM Pujanauski, L Qi, JC Kash and JK Taubenberger. (2015). An intranasal virus-like particle vaccine broadly protects mice from multiple subtypes of influenza A virus. MBio 6:e01044.

7. Fouchier RA, V Munster, A Wallensten, TM Bestebroer, S Herfst, D Smith, GF Rimmelzwaan, B Olsen and AD Osterhaus. (2005). Characterization of a novel influenza A virus hemagglutinin subtype (H16) obtained from blackheaded gulls. J Virol 79:2814-2822.

8. Cohen AL, O Hellferscee, M Pretorius, F Treurnicht, S Walaza, S Madhi, M Groome, H Dawood, E Variava, et al. (2014). Epidemiology of influenza virus types and subtypes in South Africa, 2009-2012. Emerg Infect Dis 20:11621169.

9. Dawood FS, S Jain, L Finelli, MW Shaw, S Lindstrom, RJ Garten, LV Gubareva, X Xu, CB Bridges and TM Uyeki. (2009). Emergence of a novel swine-origin influenza A (H1N1) virus in humans. N Engl J Med 360:2605-2615.

10. Uyeki TM and NJ Cox. (2013). Global concerns regarding novel influenza A (H7N9) virus infections. N Engl J Med 368:1862-1864. 
11. Yen HL and RG Webster. (2009). Pandemic influenza as a current threat. Curr Top Microbiol Immunol 333:3-24.

12. Bouvier NM and P Palese. (2008). The biology of influenza viruses. Vaccine 26:D49-D53.

13. Samji T. (2009). Influenza A: understanding the viral life cycle. Yale J Biol Med 82:153-159.

14. Yamayoshi S, M Watanabe, H Goto and Y Kawaoka. (2016). Identification of a novel viral protein expressed from the PB2 segment of influenza A virus. J Virol 90:444-456.

15. Balgi AD, J Wang, DYH Cheng, C Ma, TA Pfeifer, Y Shimizu, HJ Anderson, LH Pinto, RA Lamb, WF DeGardo and M Roberge. (2013). Inhibitors of the influenza A virus M2 proton channel discovered using a high-throughput yeast growth restoration assay. PLoS One 8:e55271.

16. Hayden FG. (2001). Newer influenza antivirals, biotherapeutics and combinations. Influenza Other Respir Viruses 7:63-75.

17. Moscona A. (2005). Drug therapy-neuraminidase inhibitors for influenza. N Engl J Med 353:1363-1373.

18. Jefferson T, V Demicheli, C Di Pietrantonj and D Rivetti. (2006). Amantadine and rimantadine for influenza A in adults. Cochrane Database Syst Rev 19:CD001169.

19. Bright RA, MJ Medina, X Xu, G Perez-Oronoz, TR Wallis, XM Davis, L Povinelli, NJ Cox and AI Klimov. (2005). Incidence of adamantane resistance among influenza A (H3N2) viruses isolated worldwide from 1994 to 2005: a cause for concern. Lancet 366:1175-1181.

20. Hurt AC, JK Holien, MW Parker and IG Barr. (2009). Oseltamivir resistance and the H274Y neuraminidase mutation in seasonal, pandemic and highly pathogenic influenza viruses. Drugs 69:2523-2531.

21. Neumann G, T Noda and Y Kawaoka. (2009). Emergence and pandemic potential of swine-origin H1N1 influenza virus. Nature 459:931-939.

22. Poland GA, RM Jacobson and IG Ovsyannikova (2009). Influenza virus resistance to antiviral agents: a plea for rational use. Clin Infect Dis 48:1254-1256.

23. Watanabe T, E Kawakami, JE Shoemaker, TJ Lopes, Y Matsuoka, Y Tomita, H Kozuka-Hata, T Gorai, T Kuwahara, et al. (2015). Influenza virus-host interactome screen as a platform for antiviral drug development. Cell Host Microbe 16:795-805.

24. Roehr B. (1998). Fomivirsen approved for CMV retinitis. J Int Assoc Physicians AIDS Care 4:14-16.

25. Crooke ST and RS Geary. (2013). Clinical pharmacological properties of mipomersen (Kynamro), a second generation antisense inhibitor of apolipoprotein B. Br J Clin Pharmacol 76:269-276.

26. Mathews DH. (2005). Predicting a set of minimal free energy RNA secondary structures common to two sequences. Bioinformatics 21:2246-2253.

27. Lenartowicz E, J Kesy, A Ruszkowska, M SoszynskaJozwiak, P Michalak, WN Moss, DH Turner, R Kierzek and E Kierzek. (2016). Self-folding of naked segment 8 genomic RNA of influenza A virus. PLoS One 11:e0148281.

28. Kierzek E and R Kierzek. (2003). The synthesis of oligoribonucleotides containing N-6-alkyladenosines and 2-methylthio-N-6-alkyladenosines via post-synthetic modification of precursor oligomers. Nucleic Acids Res 31: 4461-4471.

29. Xia TB, J SantaLucia, ME Burkard, R Kierzek, SJ Schroeder, XQ Jiao, C Cox and DH Turner. (1998). Thermodynamic parameters for an expanded nearest-neighbor model for formation of RNA duplexes with Watson-Crick base pairs. Biochemistry 37: 14719-14735.

30. Mathews DH. (2004). Using an RNA secondary structure partition function to determine confidence in base pairs predicted by free energy minimization. RNA 10:1178-1190.

31. Breen M, A Nogales, SF Baker, DR Perez, L MartínezSobrido. (2016). Replication-competent influenza A and B viruses expressing a fluorescent dynamic timer protein for in vitro and in vivo studies. PLoS One 11:e0147723.

32. Vijaykrishna D, R Mukerji and GJ Smith. (2015). RNA Virus reassortment: an evolutionary mechanism for host jumps and immune evasion. PLoS Pathog 11:e1004902.

33. Watanabe T, G Zhong, CA Russell, N Nakajima, M Hatta, A Hanson, $\mathrm{R}$ McBride, DF Burke, $\mathrm{K}$ Takahashi, et al. (2015). Circulating avian influenza viruses closely related to the 1918 virus have pandemic potential. Cell Host Microbe 15:692-705.

34. Fraser C, CA Donnelly, S Cauchemez, WP Hanage, MD Van Kerkhove, TD Hollingsworth, J Griffin, RF Baggaley, HE Jenkins, et al. (2009). Pandemic potential of a strain of influenza A (H1N1): early findings. Science 324:1557-1561.

35. Inagaki A, H Goto, S Kakugawa, M Ozawa and Y Kawaoka. (2012). Competitive incorporation of homologous gene segments of influenza A virus into virions. J Virol 86:1020010202.

36. Hatta T, K Takai, S Nakada, T Yokota and H Takaku. (1997). Specific inhibition of influenza virus RNA polymerase and nucleoprotein genes expression by liposomally endocapsulated antisense phosphorothioate oligonucleotides: penetration and localization of oligonucleotides in clone 76 cells. Biochem Biophys Res Commun 232:545-549.

37. Abe T, T Mizuta, T Hatta, N Miyano-Kurosaki, M Fujiwara, K Takai, S Shigeta, T Yokota and H Takaku. (2001). Antisense therapy of influenza. Eur J Pharm Sci 13:61-69.

38. Ge Q, M Pastey, D Kobasa, P Puthavathana, C Lupfer, RK Bestwick, PL Iversen, J Chen and DA Stein. (2006). Inhibition of multiple subtypes of influenza A virus in cell cultures with morpholino oligomers. Antimicrob Agents Chemother 50:3724-3733.

39. Gabriel G, A Nordmann, DA Stein, PL Iversen and HD Klenk. (2008). Morpholino oligomers targeting the PB1 and NP genes enhance the survival of mice infected with highly pathogenic influenza A H7N7 virus. J Gen Virol 89:939-948.

40. Lupfer C, DA Stein, DV Mourich, SE Tepper, PL Iversen and M Pastey. (2008). Inhibition of influenza A H3N8 virus infections in mice by morpholino oligomers. Arch Virol 153:929-937.

41. Zhang T, PS Zhao, W Zhang, M Liang, YW Gao, ST Yang, TC Wang, C Qin, CY Wang and XZ Xia. (2011). Antisense oligonucleotide inhibits avian influenza virus H5N1 replication by single chain antibody delivery system. Vaccine 29:1558-1564.

42. Soszynska-Jozwiak M, P Michalak, WN Moss, R Kierzek and E Kierzek. (2015). A conserved secondary structural element in the coding region of the influenza A virus nucleoprotein (NP) mRNA is important for the regulation of viral proliferation. PLoS One 10:e0141132.

43. Giannecchini S, HM Wise, P Digard, V Clausi, E Del Poggetto, L Vesco, S Puzelli, I Donatelli and A Azzi. (2011). Packaging signals in the $5^{\prime}$-ends of influenza virus PA, PB1, and PB2 genes as potential targets to develop nucleic-acid based antiviral molecules. Antiviral Res 92:64-72. 
44. Wu Y, G Zhang, Y Li, Y Jin, R Dale, LQ Sun and M Wang. (2008). Inhibition of highly pathogenic avian H5N1 influenza virus replication by RNA oligonucleotides targeting NS1 gene. Biochem Biophys Res Commun 365:369-374.

45. Duan M, Z Zhou, RX Lin, J Yang, XZ Xia and SQ Wang. (2008). In vitro and in vivo protection against the highly pathogenic $\mathrm{H} 5 \mathrm{~N} 1$ influenza virus by an antisense phosphorothioate oligonucleotide. Antivir Ther 13:109-114.

46. Giannecchini S, V Clausi, D Nosi and A Azzi. (2009). Oligonucleotides derived from the packaging signal at the $5^{\prime}$ end of the viral PB2 segment specifically inhibit influenza virus in vitro. Arch Virol 154:821-832.

47. Fujii K, M Ozawa, K Iwatsuki-Horimoto, T Horimoto and Y Kawaoka. (2009). Incorporation of influenza A virus genome segments does not absolutely require wild-type sequences. J Gen Virol 90:1734-1740.

48. Fujii K, Y Fujii, T Noda, Y Muramoto, T Watanabe, A Takada, H Goto, T Horimoto and Y Kawaoka. (2005). Importance of both the coding and the segment-specific noncoding regions of the influenza A virus NS segment for its efficient incorporation into virions. J Virol 79:3766-3774.

49. Wang H, Z Feng, Y Shu, H Yu, L Zhou, R Zu, Y Huai, J Dong, C Bao, et al. (2008). Probable limited person-toperson transmission of highly pathogenic avian influenza A (H5N1) virus in China. Lancet 371:1427-1434.

50. Ungchusak K, P Auewarakul, SF Dowell, R Kitphati, W Auwanit, P Puthavathana, M Uiprasertkul, K Boonnak, C Pittayawonganon, et al. (2005). Probable person-to-person transmission of avian influenza A (H5N1). N Engl J Med 352:333-340.

51. Fratczak A, R Kierzek and E Kierzek. (2009). LNAmodified primers drastically improve hybridization to target RNA and reverse transcription. Biochemistry 48:514-516.

52. Mathews DH, ME Burkard, SM Freier, JR Wyatt and DH Turner. (1999). Predicting oligonucleotide affinity to nucleic acid targets. RNA 5:1458-1469.

53. Lu ZJ and DH Mathews. (2008). OligoWalk: an online siRNA design tool utilizing hybridization thermodynamics. Nucleic Acids Res 36:W104-W108.

54. Arranz R, R Coloma, F Javier Chichon, J Javier Conesa, JL Carrascosa, JM Valpuesta, J Ortin and J Martin-Benito. (2012). The structure of native influenza virion ribonucleoproteins. Science 338:1634-1637.

55. Coloma R, JM Valpuesta, R Arranz, JL Carrascosa, J Ortin and J Martin-Benito. (2009). The structure of a biologically active influenza virus ribonucleoprotein complex. PLOS Pathogens 5:e1000491

56. Baudin F, C Bach, S Cusack and RWH Ruigrok. (1994). Structure of influenza-virus RNP. I. Influenza-virus nucleoprotein melts secondary structure in panhandle RNA and exposes the bases to the solvent. EMBO J 13:3158-3165.
57. Herschlag D. (1991). Implications of ribozyme kinetics for targeting the cleavage of specific RNA molecules in vivo: more isn't always better. Proc Natl Acad Sci USA 88:69216925.

58. Roberts RW and DM Crothers. (1991). Specificity and stringency in DNA triplex formation. Proc Natl Acad Sci USA 88:9397-9401.

59. Quattrone A, L Papucci, N Schiavone, E Mini and S Capaccioli. (1994). Intracellular enhancement of intact antisense oligonucleotide steady-state levels by cationic lipids. Anticancer Drug Des 9:549-553.

60. Hutchinson EC, JC von Kirchbach, JR Gog and P Digard. (2010). Genome packaging in influenza A virus. J Gen Virol 91:313-328.

61. Noda T, Y Sugita, K Aoyama, A Hirase, E Kawakami, A Miyazawa, H Sagara and Y Kawaoka. (2012). Threedimensional analysis of ribonucleoprotein complexes in influenza A virus. Nat Commun 3:639.

62. Fournier E, V Moules, B Essere, JC Paillart, JD Sirbat, C Isel, A Cavalier, JP Rolland, D Thomas, B Lina and R Marquet. (2012). A supramolecular assembly formed by influenza A virus genomic RNA segments. Nucleic Acids Res 40:2197-2209.

63. Fournier E, V Moules, B Essere, JC Paillart, JD Sirbat, A Cavalier, JP Rolland, D Thomas, B Lina, C Isel and R Marquet. (2012). Interaction network linking the human H3N2 influenza A virus genomic RNA segments. Vaccine 30:7359-7367.

64. Gavazzi C, C Isel, E Fournier, V Moules, A Cavalier, D Thomas, B Lina and R Marquet. (2013). An in vitro network of intermolecular interactions between viral RNA segments of an avian $\mathrm{H} 5 \mathrm{~N} 2$ influenza A virus: comparison with a human H3N2 virus. Nucleic Acids Res 41:1241-1254.

65. Gavazzi C, M Yver, C Isel, RP Smyth, M Rosa-Calatrava, B Lina, V Moules and R Marquet. (2013). A functional sequencespecific interaction between influenza A virus genomic RNA segments. Proc Natl Acad Sci USA 110:16604-16609.

Address correspondence to: Douglas H. Turner, PhD Department of Chemistry University of Rochester 120 Trustee Road Rochester, NY 14627-0216

E-mail:douglas.turner@rochester.edu

Received for publication April 12, 2016; accepted after revision June 27, 2016. 